\title{
Knowledge Acquisition during Exam Preparation Improves Memory and Modulates Memory Formation
}

\author{
(1)Garvin Brod, ${ }^{1}$ CUlman Lindenberger, ${ }^{1,2}$ (๑) Anthony D. Wagner, ${ }^{3,4}$ and $\odot$ Yee Lee Shing ${ }^{1,5}$ \\ ${ }^{1}$ The Center for Lifespan Psychology, Max Planck Institute for Human Development, 14195 Berlin, Germany, ${ }^{2}$ European University Institute, 50014 San \\ Domenico di Fiesole, Italy, ${ }^{3}$ Department of Psychology and ${ }^{4}$ Neurosciences Program, Stanford University, Stanford, California 94305, and ${ }^{5}$ Division of \\ Psychology, University of Stirling, Stirling FK9 4LA, United Kingdom
}

According to the schema-relatedness hypothesis, new experiences that make contact with existing schematic knowledge are more easily encoded and remembered than new experiences that do not. Here we investigate how real-life gains in schematic knowledge affect the neural correlates of episodic encoding, assessing medical students 3 months before and immediately after their final exams. Human participants were scanned with functional magnetic resonance imaging while encoding associative information that varied in relatedness to medical knowledge (face-diagnosis vs face-name pairs). As predicted, improvements in memory performance over time were greater for face-diagnosis pairs (high knowledge-relevance) than for face-name pairs (low knowledge-relevance). Improved memory for facediagnosis pairs was associated with smaller subsequent memory effects in the anterior hippocampus, along with increased functional connectivity between the anterior hippocampus and left middle temporal gyrus, a region important for the retrieval of stored conceptual knowledge. The decrease in the anterior hippocampus subsequent memory effect correlated with knowledge accumulation, as independently assessed by a web-based learning platform with which participants studied for their final exam. These findings suggest that knowledge accumulation sculpts the neural networks associated with successful memory formation, and highlight close links between knowledge acquired during studying and basic neurocognitive processes that establish durable memories.

Key words: educational technology; fMRI; hippocampus; middle temporal gyrus; prior knowledge; schema

Significance Statement

In a sample of medical students, we tracked knowledge accumulation via a web-based learning platform and investigated its effects on memory formation before and after participants' final medical exam. Knowledge accumulation led to significant gains in memory for knowledge-related events and predicted a selective decrease in hippocampal activation for successful memory formation. Furthermore, enhanced functional connectivity was found between hippocampus and semantic processing regions. These findings (1) demonstrate that knowledge facilitates binding in the hippocampus by enhancing its communication with the association cortices, (2) highlight close links between knowledge induced in the real world and basic neurocognitive processes that establish durable memories, and (3) exemplify the utility of combining laboratory-based cognitive neuroscience research with real-world educational technology for the study of memory.

\section{Introduction}

Information contained in new experiences is learned more easily when learners can relate the information to their prior knowledge (Bransford and Johnson, 1972). This long-standing observation has commonly been attributed to individuals' ability to assimilate new experiences with existing schemas. Schemas are commonly portrayed as structured associative information, representationally distributed in neocortex, that allows for more elaborative

Department of Psychology, Stanford University, Palo Alto, CA. We thank Torben Brod, Friederike Fenski, Thomas Grandy, Andreas Horn, Daniel Kutzer, and Rebekka Rust for their help in choosing the medical diagnoses; and Silvia Bunge, Thackery Brown, Attila Keresztes, Markus Werkle-Bergner, and Marlieke van Kesteren for valuable discussions.

The authors declare no competing financial interests.

Correspondence should be addressed to Dr. Yee Lee Shing, Division of Psychology, University of Stirling, Stirling FK9 4LA, UK. E-mail:yee.shing@stir.ac.uk.

DOI:10.1523/JNEUROSCI.0045-16.2016

Copyright $\odot 2016$ the authors $\quad 0270-6474 / 16 / 368103-09 \$ 15.00 / 0$ 
encoding and provides a search frame during retrieval (Bartlett, 1932; Alba and Hasher, 1983; Ghosh and Gilboa, 2014). The integration of new experiences into existing schemas during encoding is associated with activity in anterior parts of the hippocampus (HC; Ranganath and Ritchey, 2012; Poppenk et al., 2013; Schlichting et al., 2015). Connections between newly formed associations and existing schemas presumably facilitate binding in the $\mathrm{HC}$ and the integration of these associations into existing knowledge structures (van Kesteren et al., 2012; McClelland, 2013; Preston and Eichenbaum, 2013). Consistent with this framework, studies with rodents have shown that pre-existing neocortical schemas allow rapid integration of new information, as measured by accelerated HC independence for consolidation of schema-related flavor-place associations, which are absent when connected neocortical regions are blocked (Tse et al., 2007, 2011). However, evidence from human subjects is limited (for findings in the parahippocampal gyrus, see van Kesteren et al., 2014).

The human anterior $\mathrm{HC}$ receives cross-domain inputs mainly from entorhinal cortex and binds these inputs into integrated memory representations (Prince et al., 2005; Davachi, 2006; Zimmer et al., 2006; Liang et al., 2013). HC has long-range connections to a number of neocortical areas, in particular medial prefrontal cortex (mPFC) and lateral temporal regions (Poppenk et al., 2013). Both mPFC and lateral temporal regions, especially the middle temporal gyrus (MTG), are involved in the retrieval of stored conceptual knowledge (Badre and Wagner, 2007; Lau et al., 2008; Binder et al., 2009; Turken and Dronkers, 2011).

An underlying assumption of most work investigating schema effects on memory is that knowledge accumulation in a domain, for example, via formalized instruction, should lead to enhanced memorability of new information in the domain, in the sense of a "Matthew effect" (Stanovich, 1986) or cumulative advantage. However, systematic longitudinal studies are needed to strengthen a causal interpretation of the relationship between increasing knowledge and enhanced memory for episodes related to that knowledge, which have been lacking thus far.

Here, we used a real-world educational setting that involved extensive knowledge acquisition to fill this gap. Specifically, we tested a sample of medical students who used a web-based learning platform to prepare for a state-regulated final medical exam. New episodic learning was tested with a face-word associative memory task in two fMRI sessions, one 3 months before (T1) and one right after the final exam (T2). Critically, half of the faces were paired with medical diagnosis words (high relevance of schematic knowledge), while the other half were paired with first names (low relevance of schematic knowledge).

We predicted that, first, knowledge-related facilitation of episodic memory would be greater for high relevance episodes (face-diagnosis pairs) than for low relevance episodes (facename pairs). Second, we predicted that as knowledge was gained (i.e., from T1 to T2), anterior $\mathrm{HC}$ activation associated with successful memory formation would decrease. This decrease was predicted to occur in the high-relevance condition only, putatively reflecting facilitated binding due to greater schematic support. Third, we predicted an activation increase in regions associated with the representation of schematic knowledge (Lau et al., 2008; Binder et al., 2009), such as lateral temporal regions, as well as an increase in functional connectivity between anterior $\mathrm{HC}$ and lateral temporal regions across time (T1-T2), particu- larly for the high-relevance condition. Finally, we examined whether any of these predicted changes would be related to individual differences in knowledge increase from $\mathrm{T} 1$ to $\mathrm{T} 2$, as independently assessed by the web-based learning platform.

\section{Materials and Methods}

\section{Participants}

Thirty-five medical students (20 women; age range, 23-29 years; mean age, 25.9 years) who gave written informed consent participated in the study. All participants were right-handed and had no history of psychiatric or neurological disorders. Recruitment took place via e-mails sent out to Berlin-based users of the commercial web-based learning platform AMBOSS, which prepares medical students for their final exam. Participants were paid $€ 76$. Two participants did not return for the second (T2) measurement; data from two further participants were excluded because they did not make proper use of the memory confidence scale (see below), leaving too few remembered trials $(n \leq 10)$ for analysis. We thus analyzed data from 31 participants. The study also included a control group (CG) of 16 medical students (mean age, 25.0 years), whose final exam lay $0.5-1$ year ahead of them, to assess changes in brain structure (not reported in this article, but see behavioral results of the memory task section). The CG participants were also tested twice in the course of 3 months, but did not study intensively during this time (which was confirmed via questionnaires), and did not use the web-based learning platform. Ethics approval was obtained from the ethics committee of the German Psychological Society.

\section{General design and procedure}

Participants were tested twice, once 3 months before their final stateregulated written medical exam (T1), and again shortly after (mean interval, $11.7 \mathrm{~d}$; range, 1-22 d) their written exam (T2). In between, they intensively prepared for the exam using the web-based learning platform. The exam takes $3 \mathrm{~d}(5 \mathrm{~h} / \mathrm{d})$ and consists of a total of 320 multiple-choice questions, covering all clinical subjects taught during the final 3 years of medical school in Germany. On the learning platform, they followed a structured $100 \mathrm{~d}$ learning plan that guided them through all of the topics relevant for the final exam. Each learning day consisted of solving exam questions from previous years and reading relevant information (e.g., on symptoms, etiology, epidemiology, pathophysiology) about the medical syndromes and diagnoses covered in the questions. Exam questions were multiple-choice questions with five response options, of which only one was correct. Participants received detailed feedback on their responses. We stress that, although the web-based learning platform was critical to our study design because it allowed close monitoring of knowledge accumulation in our sample, our design does not permit any conclusions about the effectiveness of studying with it compared to other methods of studying.

Measuring learning performance and success. We measured participants' learning performance using data provided by the learning platform. We focused on daily measures of the number of questions answered on the platform, and the correctness of the answers (percentage correct) as a measure of their knowledge. To measure the increase in medical knowledge from T1 to T2, we first calculated, for each individual, the average accuracy (percentage correct) for answered questions during the first week of studying on the learning platform (T1 measurement). This score was subtracted from the average percentage correct score achieved during the week before the real exam (T2 measurement), in which participants performed mock exam consisting of previously nonstudied questions.

Because the medical students differed in their initial level of knowledge, with some students having high knowledge already at T1 and others lower knowledge, and because there is a finite number of old exam questions, participants' change in accuracy (T2-T1) can be expected to correlate negatively with accuracy at $\mathrm{T} 1$. This was indeed the case, such that participants who started with a high level of knowledge showed less change in accuracy $(r=-0.82, p<0.001)$. To control for this negative correlation between change and initial performance on the platform, we used a residualized change score (Cohen et al., 2003). To obtain a validity 

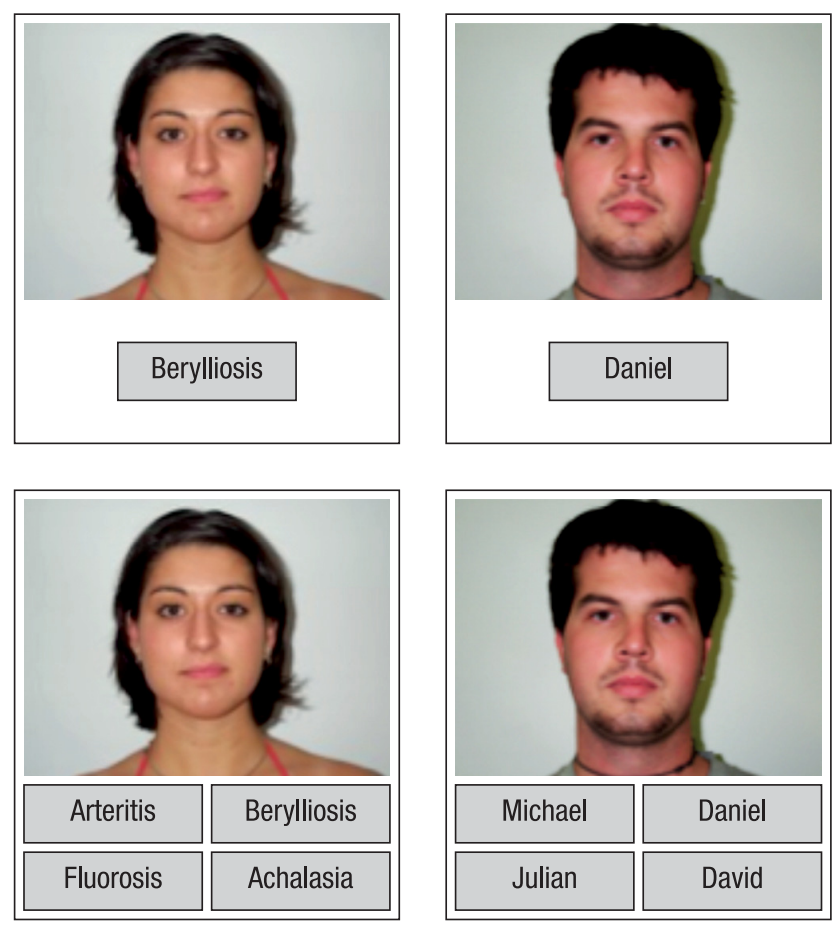

Figure 1. Memory task. In the MR scanner (upper panel), participants memorized faceword pairs, which were presented for $5 \mathrm{~s}$ each. Half of the words were diagnoses (left example) and half were first names (right example). Outside of the scanner (lower panel), participants were presented with all of the studied faces, together with four first name or four diagnosis options, of which only one had been presented with the face during the encoding phase. The other three were familiar names or diagnoses that had been paired with other faces. The participants' task was to select the option that had been presented with the face during encoding.

check of our learning measures, we asked the participants to report their final exam scores.

Memory stimuli, task, and behavioral analyses. The encoding phase took place in the MRI scanner, and participants were instructed to memorize 140 face-word pairs, in which half of the words were diagnoses and the other half were first names (Fig. 1). We predicted that encoding of a face-diagnosis association would activate a medicine-related network of schematic knowledge in the participants, and that this network would increase in strength and connection over the period of intensive learning. Over time, this should then lead to a differential encoding advantage for face-diagnosis over face-name pairs. Two parallel stimulus lists were created to allow counterbalancing across participants and sessions. A total of 140 medical diagnoses and 140 common German first names were used together with 140 neutral face pictures. Each face was pseudorandomly combined with one diagnosis and one name. Thus, while participants saw each face twice, once at T1 and once at T2, they saw each diagnosis and name only once during the whole study. To ensure that our diagnosis stimuli were sensitive to change in knowledge, they were chosen from a wide array of diagnoses relevant for the final exam based on a rating by four recent medical graduates regarding their difficulty and prevalence. Based on these ratings, highly frequent diagnoses (e.g., hypertension) as well as highly similar diagnoses (e.g., type 1 diabetes vs type 2 diabetes) were discarded. The face stimuli consisted of pictures of Caucasian young adults taken from the Center for Vital Longevity Face Database (Minear and Park, 2004). Faces and names were matched for gender. To avoid highly implausible face-diagnosis pairs, these pairs were matched for sex (e.g., pre-eclampsia was used only for female faces) as well as for age specificity (only diagnoses that can affect young adults were chosen). Face-diagnosis and face-name pairs were presented for $5 \mathrm{~s}$ each in an interleaved fashion (in pseudorandom order). Trials were separated by a variable fixation cross period of $2-5 \mathrm{~s}$ (mean, $3.5 \mathrm{~s}$ ). During each session (T1 or T2), there were two experimental blocks, each consisting of 70 trials.
Before entering the scanner, participants were instructed to try to memorize both the face-diagnosis and face-name pairs equally well, and were told that there would be a memory test outside of the scanner. To ensure that the participants were paying attention to the task and to promote elaborative encoding, they were asked to indicate for each faceword pair whether or not the name/diagnosis fit with the face, responding with their left/right index finger. Left/right response options were counterbalanced across participants. The encoding phase took $20 \mathrm{~min}$ in total and was performed after the structural scans.

The retrieval phase took place outside of the scanner, $\sim 10 \mathrm{~min}$ after the end of the encoding session. Participants were presented with all 140 faces again in a pseudorandom order. For each face, they were given four first names or four diagnoses, of which one had been presented with the face during the encoding phase (target), whereas the other three were names/diagnoses seen with other faces during encoding (lures). Participants indicated their choice via button press. Afterward, they were asked to indicate their decision confidence on a scale of 1 (guess) to 4 (very sure). They were given no time limit for their responses, but were told to answer as quickly and as correctly as possible.

Data were analyzed using R ( $\mathrm{R}$ Core Team, 2014). A repeatedmeasures ANOVA was performed with condition (diagnoses/names) and time (T1, T2) as within-subject factors to test for differences in memory (percentage of correctly retrieved associations, independent of decision confidence).

fMRI data acquisition and preprocessing. T2*-weighted echoplanar images (EPIs) were acquired using a 3 T Siemens TIM Trio MRI scanner [direction, transverse (interleaved ascending); FOV, 216 mm; TR, 2500 $\mathrm{ms}$; TE, $30 \mathrm{~ms}$; number of slices, 45 ; slice thickness, $2.5 \mathrm{~mm}$; matrix, $72 \times$ 72 ; voxel size, $3 \times 3 \times 2.5 \mathrm{~mm}$; distance factor, $20 \%$; two runs with 232 volumes each, including four dummy volumes each). To attenuate signal dropout in orbitofrontal regions, the slice orientation was tilted upward vertically by $15^{\circ}$ after alignment to the anterior commissure-posterior commissure plane (Weiskopf et al., 2006). To estimate geometric distortion and signal loss in the EPI, an additional $53 \mathrm{~s}$ fieldmap was acquired. Structural data were acquired using a T1-weighted 3D magnetizationprepared rapid gradient echo sequence (TR, $2500 \mathrm{~ms}$; TE, $2500 \mathrm{~ms}$; sagittal orientation; spatial resolution, $1 \times 1 \times 1 \mathrm{~mm}$ ).

Data were preprocessed and analyzed using FEAT in FSL [FMRIB Software Library (http://www.fmrib.ox.ac.uk/fsl); Smith et al., 2004]. Functional data were corrected for motion (MCFLIRT), slice acquisition times (interleaved), and local field inhomogeneities (BBR/FUGUE), then high-pass filtered $(80 \mathrm{~Hz})$ and spatially smoothed using a $5 \mathrm{~mm}$ fullwidth half-maximum Gaussian filter, resulting in a final estimated spatial smoothness of $6.9 \times 6.8 \times 6.6 \mathrm{~mm}^{3}$. Data were first coregistered with the structural image and then spatially normalized into a common space [Montreal Neurological Institute (MNI) 152 standard space, $2 \mathrm{~mm}^{3}$ ].

\section{fMRI analyses}

Brain activation. First-level analyses were conducted for individual participants, separately for the two runs at $\mathrm{T} 1$ and at $\mathrm{T} 2$. Using general linear modeling (GLM), regressors were generated by convolving the impulse function related to the onset and length of encoding events with a gamma hemodynamic response function ( $5 \mathrm{~s}$ boxcar function). Using behavioral data from retrieval, we sorted encoding trials according to their later memory outcome to investigate subsequent memory effects (SMEs, remembered > forgotten contrast; Brewer et al., 1998; Wagner et al., 1998; Paller and Wagner, 2002). Five types of events were modeled with separate regressors in the GLM. Trials that received a correct retrieval response with a confidence rating of $>1$ (i.e., nonguessing trials) were classified as remembered diagnosis or remembered name event; those that received an incorrect response were classified as forgotten diagnosis or forgotten name event. A fifth regressor of no interest was included for all remembered events that received "guessing" ratings on the confidence scale. Overall, the number of correct guesses was low (mean_T1/T2 = 8.9/8.6 of 140 trials across the two runs) and was higher for the name (mean_T1/T2 $=13.3 / 12.7)$ than for the diagnosis (mean_T1/T2 $=4.5 /$ $4.4)$ condition $\left(\mathrm{T} 1: t_{(30)}=5.50, p<0.001 ; \mathrm{T} 2: t_{(30)}=5.49, p<0.001\right)$. This condition difference was due to the stronger tendency to give a guess rating for the name (mean_T1/T2 $=38.7 / 34$ ) than for the diagnosis 

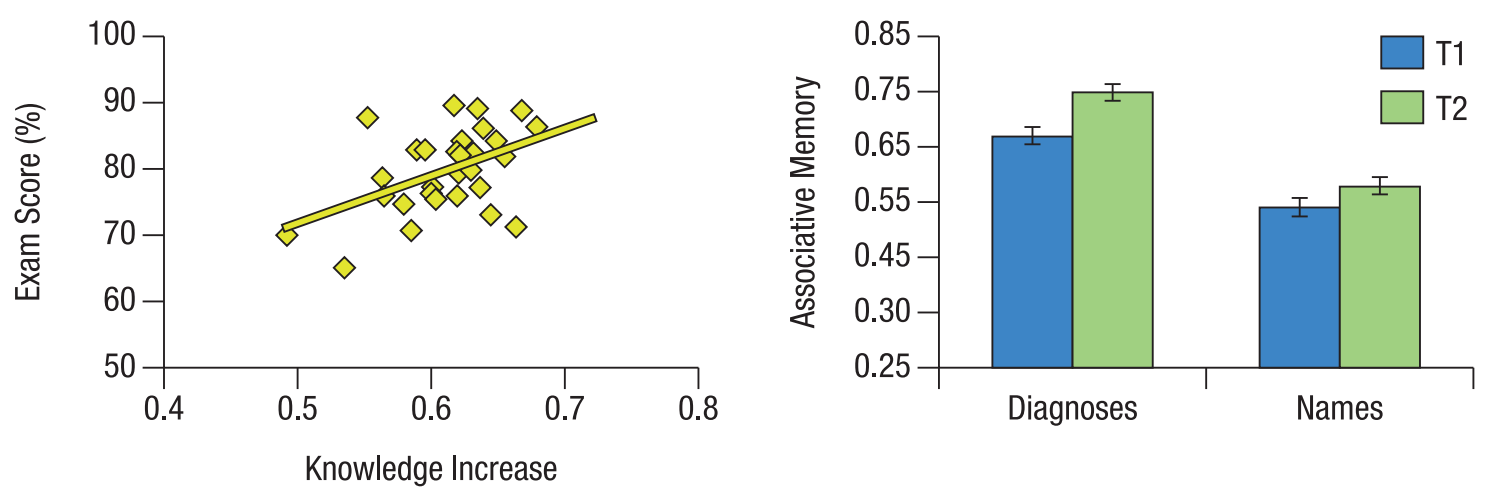

Figure 2. Correlation between medical knowledge gains and final exam score; memory performance. Left, Gains in medical knowledge, assessed via the web-based learning platform, correlated with the final exam score $(r=0.53, p<0.001)$. Right, Gains in associative memory performance were more pronounced for face- diagnosis pairs than for face-name pairs. SEs reflect the pooled error term of the within-subjects $F$ statistic.

(mean_T1/T2 $=12.6 / 9.8)$ condition $\left(\mathrm{T} 1: t_{(30)}=7.09, p<0.001 ; \mathrm{T} 2: t_{(30)}\right.$ $=6.58, p<0.001)$. SMEs, defined by the remembered $>$ forgotten contrast, were computed for the face-diagnosis and face-name conditions separately.

In a next step, the two runs were combined using a within-subjects fixed-effects analysis and normalized into MNI space. To test for changes in brain activation from $\mathrm{T} 1$ to $\mathrm{T} 2$ that differed by condition (diagnosis, name), a within-subjects fixed-effects analysis was performed that tested for differences in SME between time points $(\mathrm{T} 1, \mathrm{~T} 2)$ that were larger for the diagnoses than for the names, and vice versa (memory $\times$ time $\times$ condition interaction). To better understand any significant pattern observed in the memory $\times$ time $\times$ condition interaction, additional within-subjects fixed-effects analyses were performed to test for differences in the SME between T1 and T2 for each of the conditions separately. Across-subjects analyses were performed using a mixedeffects model in the FLAME framework in FSL. The $z$-statistic images were thresholded voxelwise at a threshold of $z>2.3$. A multiplecomparison correction was performed using the 3DClustSim program of the AFNI software package (version 16.0.04; http://afni.nimh.nih.gov/ pub/dist/doc/program_help/3dClustSim.html), which computes minimum cluster-extent thresholds for specific regions of interest using Monte Carlo simulation analysis. In addition to a whole-brain gray matter mask, based on our a-priori hypothesis about changes in the anterior $\mathrm{HC}$, we created an anatomical mask of the bilateral anterior $\mathrm{HC}$ using the probabilistic Harvard-Oxford Subcortical Structural Atlas, including voxels located at the anterior $35 \%$ of the long axis of the $\mathrm{HC}$ and with at least $25 \%$ probability of being inside the HC. Smoothness of our group-level data was estimated on the residual time series image using the AFNI 3dFWH Mx (http://afni.nimh.nih.gov/pub/dist/doc/program_help/3dFWHMx. $\mathrm{html}$ ). A simulation with 10,000 runs yielded minimum cluster extents of 17 (HC) and 143 (whole-brain) voxels to maintain a familywise error rate of $p<0.05$.

To determine the source of the observed change in SME in the anterior $\mathrm{HC}$ for the diagnosis condition, and to find out whether this change was related to individual differences in knowledge increase (as measured by behavioral performance during the first and last week of studying on the learning platform), the percentage signal change was extracted from the memory $\times$ time interaction contrast and correlated with the residualized change score.

Brain connectivity. We tested for changes in coupling between the anterior $\mathrm{HC}$ and lateral temporal regions using psychophysiological interaction (PPI) analysis separately for the left and right HC. The time courses of left and right anterior HC (using an anatomical mask, as described in the previous section) served as the physiological regressor in the model. Psychological regressors were defined as representing the remembered $>$ forgotten (SME) contrast (i.e., remembered - forgotten, and in addition remembered + forgotten to model the shared variance), which were convolved with a Gamma hemodynamic response function. Finally, a PPI regressor representing the interaction of the psychological and the physiological regressors was created. The three regressor types were then added to the existing brain activation GLM, replacing the corresponding remembered and forgotten events. This was done separately for the two conditions, runs, and time points, which were then combined at higher levels using within-subjects fixed-effects analyses and between-subjects mixed-effects models in the same way as the activation analyses. In an additional analysis, to test whether the observed connectivity changes were related to changes in knowledge, the residualized gain score was entered as a covariate into the between-subjects mixed-effects model.

\section{Results}

\section{Knowledge accumulation predicts final exam score}

During the $100 \mathrm{~d}$ of intensive studying, participants answered on average 7460 questions (range, 3702-10605 questions; SD, 1818.19 questions) from earlier exams; thus, on average 75 questions/d (range, 0-440 questions/d; SD, 17.15 questions/d). During the first week of studying at our T1 measurement, $69 \%$ (range, $48-83 \%$; SD, 9.6\%) of the earlier exam questions were answered correctly. During the wrap-up period in the last week before the real final exam, $83 \%$ of the questions (range, 69-92\% of the questions; $\mathrm{SD}, 5.4$ ) were answered correctly (change in performance on answering questions: $t_{(30)}=10.02, p<0.001$ ). The mean performance on the actual written final exam was $80 \%$ (range, 65-90\%; SD, 6.4). The total number of old exam questions studied during the $100 \mathrm{~d}$ was positively related to the final exam score $(r=0.41, p=0.01)$. Importantly, the residualized gain in knowledge (first to last week) correlated with the final exam score ( $r=0.47, p=0.005$; Fig. 2 , left). In sum, during the $100 \mathrm{~d}$, participants used the learning platform extensively and substantially increased their medical knowledge, which was measured by the change in performance between the first and last week of studying. Furthermore, performance on the learning platform was highly predictive of their exam success later on.

\section{Enhanced memory improvement for knowledge-related information}

A repeated-measures ANOVA revealed the following: (1) a main effect of condition, indicating better memory for diagnoses compared with names $\left(F_{(1,30)}=75.24, p<0.001\right)$; (2) a main effect of time, indicating improved memory performance at $\mathrm{T} 2\left(F_{(1,30)}=\right.$ 8.24, $p=0.01)$; and (3) a marginally significant interaction $\left(F_{(1,30)}=3.83, p=0.059\right)$, suggesting a greater increase in memory for face-diagnosis pairs than for face-name pairs (Fig. 2, right). In addition, to confirm that this enhanced memory improvement for face-diagnosis pairs in the exam candidates (ECs) was specific to increased medical knowledge, we compared it to a 
a

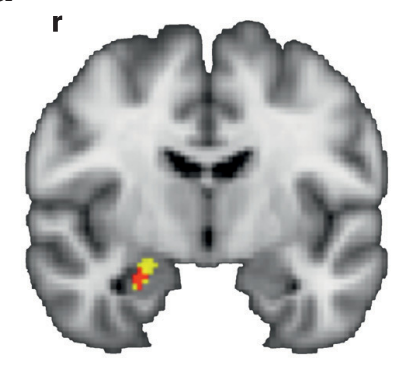

b

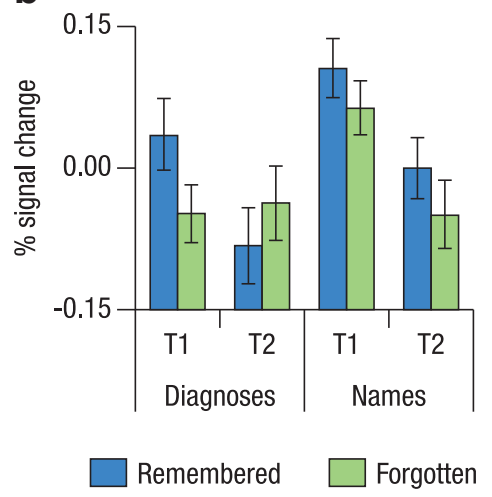

C

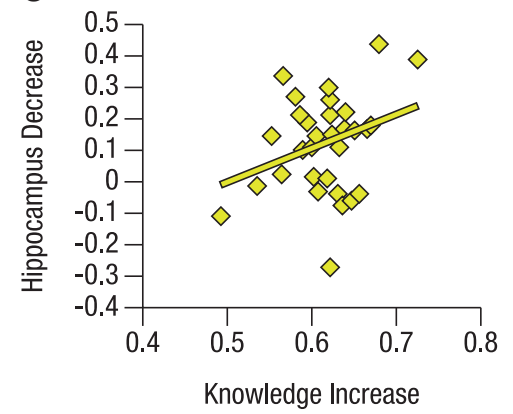

Figure 3. Time $\times$ condition interaction for SME in the right anterior HC. $\boldsymbol{a}$, Reductions in the SME from pretest to posttest were larger for face- diagnosis pairs than for face-word pairs in a cluster of voxels in the right anterior HC (in red; peak voxel: $26,-8,-20$; overlaid on the memory $\times$ time interaction for face-diagnosis pairs in yellow). $\boldsymbol{b}$, Percentage signal change of the time $\times$ condition interaction for SME in the right anterior HC. While the difference between subsequently remembered and forgotten events remained comparable across the two time points for the names condition, for the diagnosis condition there was a significant memory $\times$ time interaction. This interaction was driven by the remembered trials that displayed decreased anterior $\mathrm{HC}$ activation. $c$, Decrements of the SME for face- diagnosis pairs in this cluster of voxels correlated with individual differences in medical knowledge gains $(r=0.32, p=0.04)$.

CG of medical students who did not study intensively during this period. At T1, memory performance was similar between the two groups for the high knowledge-relevance condition (EC: $67.8 \%$, $\left.\mathrm{SD}=11.5 ; \mathrm{CG}: 67.1 \%, \mathrm{SD}=9.2 ; t_{(45)}=0.21, p=0.83\right)$ and for the low knowledge-relevance condition (EC: $54.6 \%$, SD = 13.6; CG: $\left.49.1 \%, \mathrm{SD}=10.7 ; t_{(45)}=1.4, p=0.17\right)$. At T2, memory performance for the high knowledge-relevance condition was better in the exam candidates (EC: $75.2 \%, \mathrm{SD}=11.9$; CG: $68.1 \%$, $\left.\mathrm{SD}=13.9 ; t_{(45)}=1.83, p=0.037\right)$, but again was similar for the low knowledge-relevance condition (EC: $58.1 \%$, SD = 15.5; CG: $\left.58.2 \%, \mathrm{SD}=12.4 ; t_{(45)}=0.02, p=0.98\right)$. A three-way mixed ANOVA revealed a reliable group $\times$ condition $\times$ time interaction $\left(F_{(1,45)}=11.44, p=0.001\right)$. In particular, the EC group $(\Delta$, $+7.2 \%$; SE, 0.017$)$ showed a greater increase in memory for the high knowledge-relevance condition than the CG $(\Delta,+1.1 \%$; SE, $0.028)$ from T1 to T2 $\left(t_{(45)}=2.0, p=0.02\right.$, one-tailed $)$. For the low knowledge-relevance condition, the CG $(\Delta,+9.1 \%$; SE, 0.027 ) showed a numerically greater increase in memory than the EC group $(\Delta,+3.5 \%$; SE, 0.024$)$ from T1 to T2, which did not reach significance $\left(t_{(45)}=1.4, p=0.16\right.$, two-tailed).

\section{Knowledge accumulation is associated with decreased} hippocampal activation during successful memory formation At the neural level, we found a significant memory $\times$ time $\times$ condition interaction. Specifically, a cluster in the right anterior HC (peak voxel: 26, $-8,-20$; Fig. $3 a$ ) showed an acrosstime decrease in the SME that was larger for face-diagnosis pairs (high knowledge-relevance) than for face-name pairs (low knowledge-relevance). Follow-up analyses for the two conditions separately (memory $X$ time) showed a decrease in SME from T1 to T2 for face-diagnosis pairs in a cluster in the right anterior HC (peak voxel: 26, $-6,-22$ ) overlapping with the cluster identified in the three-way interaction; no significant decrease in SME across time was observed for the face-name pairs. Thus, the memory $\times$ time $\times$ condition interaction was driven by a decrease in SME for the condition with high knowledgerelevance (for illustration, see extracted percentage signal change). Testing for increases in SME for either condition did not yield any significant effects. No decreases in SME were detected outside of the $\mathrm{HC}$ for either condition.

To examine whether the observed right $\mathrm{HC}$ T1-T2 decrease in the SME for face-diagnosis pairs was related to individual differ- ences in medical knowledge increase, the percentage signal change was extracted from the memory $\times$ time interaction cluster in the right anterior $\mathrm{HC}$ and correlated with the residualized gain between the first and last week of studying on the learning platform. This analysis revealed a significant positive correlation between knowledge increase and hippocampal SME decrease $(r=0.32, p=0.04$; Fig. 3, right).

\section{Knowledge accumulation is associated with increased hippocampus-neocortex connectivity during successful memory formation}

PPI analyses were performed to assess whether the anterior HC showed differential changes in connectivity with neocortical areas for the two conditions. For the right $\mathrm{HC}$ seed, testing for regions where across-time increases in functional connectivity for subsequently remembered versus forgotten episodes (i.e., SME in connectivity) are more pronounced for the face-diagnosis condition than for the face-name condition revealed no significant effects. However, we observed several sizable neocortical clusters with voxels just below the significance threshold $(z>$ 2.3). Given that PPI contrasts have less statistical power than activation contrasts, which is due to multicollinearities between the interaction term and the psychological and physiological terms (O'Reilly et al., 2012), we performed an additional test at a lowered voxel threshold of $z>1.96$. To account for the lower voxel threshold for multiple-comparison correction, we performed another Monte Carlo simulation using 3DClustSim, which yielded a minimum cluster extent of 497 voxels (whole brain) to maintain a familywise error rate of $p<0.05$. This analysis revealed a significant memory $\times$ time $\times$ condition interaction in the left posterior MTG (peak: $-60,-48,8$; Fig. 4). To follow up, we tested for across-time increases in functional SME connectivity separately for face-diagnosis and face-name conditions (note: voxel threshold, $z>2.3$; cluster threshold, $p<0.05$ ). The follow-up analyses showed that the observed three-way interaction reflected a specific increase for the face-diagnosis condition, as indicated by an overlapping cluster in left posterior MTG (peak: $-60,-48,8$ ), which was not present for the facename condition. In addition, both conditions displayed an extensive network of neocortical regions with greater functional connectivity to the $\mathrm{HC}$ seed at T2 than T1 (for an overview of the results, see Table 2). Testing for regions where the time-related 
a

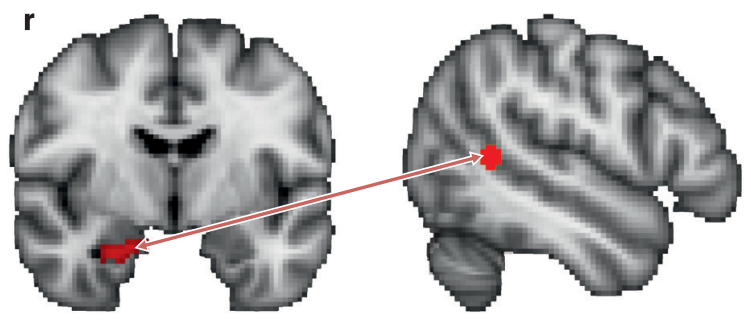

b

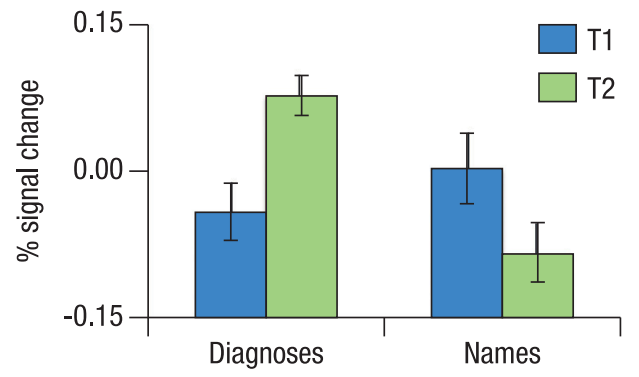

Figure 4. $\quad \boldsymbol{a}$, Time $\times$ condition interaction for SME in functional connectivity (PPI) between the right anterior hippocampus and the left middle temporal gyrus. $\boldsymbol{b}$, Using the right anterior hippocampus as a seed, we found a significant time $\times$ condition interaction with the left MTG, which was driven by a stronger increase for face- diagnosis pairs compared with face-name pairs.

increase in SME was larger for the face-name condition revealed no significant effects. In addition, changes in connectivity were not related to gains in knowledge.

For the left HC seed, testing for regions where the time-related increase in SME was larger for face-diagnosis pairs than for facename pairs (voxel threshold, $z>2.3$; cluster threshold, $p<0.05$ ) yielded two clusters in the left and right lingual gyrus/temporaloccipital fusiform cortex (peaks: $14,-64,2 ;-34,-40,-6$, respectively). Follow-up tests for PPI increases from T1 to T2 yielded a highly similar network to the one observed for the right HC, including left MTG $(-58,-48,8)$, for the face-diagnosis condition only, as well as an extensive network of neocortical regions for both conditions (see Table 2). Again, no enhanced increases in SME were observed for the face-name condition and changes in connectivity were not related to gains in knowledge.

\section{Discussion}

By following a group of medical students who studied for their final medical exam, we were able to show that an increase in schematic knowledge induced by 3 months of intensive studying was associated with gains in memory for knowledge-related episodic events. These gains were further associated with a selective decrease in SME in the right anterior HC during encoding. This decrease was related to individual differences in the accumulation of knowledge, as measured by participants' performance on the learning platform. Furthermore, we observed an increase in connectivity SME between the anterior $\mathrm{HC}$ and the left posterior MTG, a brain area that is key to semantic processing (Badre and Wagner, 2007; Hickok and Poeppel, 2007; Binder et al., 2009; Turken and Dronkers, 2011). For the first time, our study demonstrates close links between changes in knowledge induced in a real-world educational setting and changes in encoding-related brain activation as observed with a laboratory memory paradigm.

Prior knowledge facilitates the acquisition of new, related information, presumably because it provides a pre-existing associative network that offers many links to which the new information can be bound and assimilated (Piaget, 1952; van Kesteren et al., 2012; Brod et al., 2013; Ghosh and Gilboa, 2014). Recently, the notion that binding in the $\mathrm{HC}$ is facilitated by the presence of a schema has received increased attention (Wang and Morris, 2010; van Kesteren et al., 2012; McClelland, 2013; Preston and Eichenbaum, 2013), mainly sparked by a study that showed accelerated consolidation (HC independence) of schema-related information in rodents (Tse et al., 2007). In humans, this facilitation due to schema is expected to manifest itself as a decrease in $\mathrm{HC}$ activation during successful memory formation; that is, a decrease in the difference in $\mathrm{HC}$ activation for later remembered versus forgotten events (Zeithamova et al., 2012; van Kesteren et
Table 1. Regions exhibiting stronger activation at $\mathrm{T} 1$ for later remembered compared with later forgotten face-word pairs

\begin{tabular}{|c|c|c|c|c|c|}
\hline \multirow[b]{2}{*}{ Region } & \multicolumn{3}{|c|}{ MNI coordinates } & \multirow{2}{*}{$\begin{array}{l}\text { Voxels } \\
(n)\end{array}$} & \multirow[b]{2}{*}{$z-\operatorname{Max}$} \\
\hline & $x$ & $y$ & $z$ & & \\
\hline \multicolumn{6}{|c|}{ Subsequent memory effect (rem > forg) at T1 } \\
\hline Right lateral occipital cortex & 34 & -88 & 18 & 3030 & 4.25 \\
\hline Left inferior temporal gyrus & -40 & -54 & -18 & 2116 & 4.69 \\
\hline Left inferior frontal gyrus/frontal pole & -42 & 42 & -6 & 1662 & 3.91 \\
\hline Bilateral superior frontal gyrus & -2 & 52 & 44 & 1381 & 4.69 \\
\hline Right hippocampus/amygdala & 20 & -6 & -14 & 1010 & 4.27 \\
\hline Left hippocampus/amygdala & -16 & -4 & -12 & 509 & 3.88 \\
\hline Bilateral ventromedial PFC & -4 & 48 & -14 & 401 & 3.73 \\
\hline Left lateral occipital cortex & -48 & -70 & 36 & 310 & 3.55 \\
\hline Right inferior frontal gyrus & 56 & 34 & 12 & 256 & 3.60 \\
\hline Right precentral gyrus & 48 & 8 & 30 & 221 & 3.79 \\
\hline Left superior frontal gyrus & -6 & 20 & 54 & 187 & 3.16 \\
\hline \multicolumn{6}{|c|}{ Diagnosis (rem $>$ forg) $>$ Name (rem $>$ forg) at T1 } \\
\hline Right angular gyrus & 54 & -48 & 28 & 246 & 3.37 \\
\hline Frontal pole & 32 & 64 & 6 & 220 & 3.31 \\
\hline Right paracingulate gyrus & 8 & 48 & 26 & 169 & 2.90 \\
\hline Bilateral precuneus & -2 & -58 & 62 & 158 & 3.13 \\
\hline Right middle temporal gyrus & 70 & -28 & -8 & 148 & 3.36 \\
\hline \multicolumn{6}{|c|}{ Name (rem $>$ forg) $>$ Diagnosis (rem $>$ forg) at T1 } \\
\hline Left lateral occipital cortex & -42 & -78 & -6 & 382 & 3.28 \\
\hline Left precentral gyrus & -46 & -4 & 46 & 227 & 3.63 \\
\hline Right lateral occipital cortex & 38 & -58 & -6 & 224 & 3.78 \\
\hline
\end{tabular}

Regions exhibiting stronger activation at T1 for later remembered as compared to later forgotten diagnoses or names (Subsequent memory effect, top). Regions exhibiting a stronger subsequent memory effect for diagnoses than for names, and vice versa, at T1 (condition $\times$ memory interaction, bottom). Voxel threshold, $z>2.3$; cluster threshold, $p<0.05$. rem, Remembered; forg, forgotten.

al., 2012). To date, empirical evidence for this prediction with human subjects has been limited, except for some hints from a study by Zeithamova et al. (2012), showing that increased activation in mPFC, coupled with decreased activation in $\mathrm{HC}$ across study episodes within a session, predicted successful inference. This decrease in HC activity across study repetitions was taken to reflect either progressively more efficient coding in the $\mathrm{HC}$ or a decreased need for binding due to stronger overlaps with earlier events (Zeithamova et al., 2012). Indeed, comparing HC SME between the face-diagnosis pairs and face-name pairs during our $\mathrm{T} 1$ measurement did not reveal any HC differences. Both conditions yielded strong anterior HC activity (Table 1). Arguably, due to the HC being highly active during successful encoding of both schema-related and schema-unrelated information, condition differences in activation that are due to varying levels of schema knowledge are typically too subtle to be reliably measured by a one-occasion fMRI research design. In contrast, comparisons over time within individuals whose knowledge base is expanding may be more sensitive to capturing the decreasing relationship between successful encoding and activation magnitude in the 
Table 2. Regions exhibiting increases in functional connectivity with the hippocampus for subsequently remembered versus forgotten face-word pairs

\begin{tabular}{|c|c|c|c|c|c|}
\hline \multirow[b]{2}{*}{ Region } & \multicolumn{3}{|c|}{ MNI coordinates } & \multirow{2}{*}{$\begin{array}{l}\text { Voxels } \\
(n)\end{array}$} & \multirow[b]{2}{*}{$z-M a x$} \\
\hline & $x$ & $y$ & $z$ & & \\
\hline \multicolumn{6}{|l|}{ Seed: right hippocampus } \\
\hline \multicolumn{6}{|l|}{$\begin{array}{l}\text { Face- diagnosis condition: } \mathrm{T} 2 \text { (rem }>\text { forg) }>\mathrm{T} 1 \\
\quad(\text { rem }>\text { forg })\end{array}$} \\
\hline Left middle/inferior frontal gyrus & -38 & 40 & 30 & 1498 & 4.36 \\
\hline Left insular cortex & -34 & -16 & 14 & 1004 & 3.83 \\
\hline Right superior frontal gyrus & 2 & 54 & 40 & 772 & 3.56 \\
\hline Right frontal pole/medial prefrontal cortex & 34 & 38 & -6 & 695 & 4.16 \\
\hline Right lateral occipital cortex & 58 & -62 & 6 & 478 & 3.7 \\
\hline Left middle temporal gyrus & -60 & -48 & 8 & 440 & 4.09 \\
\hline Right lingual gyrus & 14 & -66 & 2 & 352 & 3.51 \\
\hline Right postcentral gyrus & 16 & -34 & 74 & 181 & 3.35 \\
\hline Right temporal pole & 50 & 12 & -12 & 160 & 3.52 \\
\hline Right putamen & 28 & 10 & -6 & 158 & 3.45 \\
\hline Left postcentral gyrus & -10 & -36 & 78 & 149 & 3.42 \\
\hline \multicolumn{6}{|l|}{$\begin{array}{l}\text { Face-name condition: T2 (rem }>\text { forg) }>\text { T1 } \\
\qquad \text { (rem }>\text { forg) }\end{array}$} \\
\hline Left frontal pole/medial prefrontal cortex & -6 & 56 & 6 & 1999 & 4.13 \\
\hline Left postcentral gyrus & -60 & -22 & 20 & 215 & 3.8 \\
\hline Left postcentral gyrus & -50 & -12 & 28 & 191 & 3.58 \\
\hline Right central opercular cortex & 60 & 4 & 2 & 166 & 3.45 \\
\hline Left precuneus & -6 & -58 & 42 & 150 & 3.41 \\
\hline Right precentral gyrus & 64 & 6 & 26 & 148 & 3.67 \\
\hline \multicolumn{6}{|l|}{ Seed: left hippocampus } \\
\hline \multicolumn{6}{|c|}{$\begin{array}{l}\text { Face-diagnosis condition: T2 (rem }>\text { forg) }>\text { T1 } \\
\text { (rem }>\text { forg) }\end{array}$} \\
\hline Left frontal pole/inferior frontal gyrus & -38 & 44 & -4 & 1975 & 4.13 \\
\hline Bilateral superior/medial prefrontal cortex & -2 & 58 & 26 & 357 & 3.28 \\
\hline Medial prefrontal cortex & -10 & 38 & -10 & 354 & 3.25 \\
\hline Left middle temporal gyrus & -58 & -48 & 8 & 317 & 3.53 \\
\hline Right frontal pole & 24 & 56 & 28 & 179 & 3.19 \\
\hline Left posterior hippocampus & -32 & -32 & -6 & 147 & 3.66 \\
\hline \multicolumn{6}{|l|}{$\begin{array}{l}\text { Face-name condition: } \mathrm{T} 2 \text { (rem }>\text { forg) }>\mathrm{T} 1 \\
\qquad \text { (rem }>\text { forg) }\end{array}$} \\
\hline Left frontal pole/medial prefrontal cortex & -8 & 50 & -6 & 1190 & 3.82 \\
\hline Left precuneus & -8 & -60 & 42 & 299 & 3.56 \\
\hline Right central opercular cortex & 58 & -8 & 12 & 286 & 3.43 \\
\hline Right frontal pole & 8 & 54 & 32 & 203 & 3.14 \\
\hline Left frontal pole & -36 & -48 & 18 & 150 & 3.28 \\
\hline
\end{tabular}

Regions exhibiting increases in functional connectivity with the left and right anterior hippocampus, respectively, for subsequently remembered versus forgotten episodes (i.e., SME in connectivity). Voxel threshold, $z>2.3$; cluster threshold, $p<0.05$. rem, remembered; forg, forgotten.

anterior HC. Hence, we conclude that extending one's knowledge base through the acquisition of schematic knowledge facilitates binding in the $\mathrm{HC}$, presumably by increasing the number of potential associative links to the knowledge base.

Contrary to expectations, we did not find increased SME in lateral temporal regions across the two time points for the high knowledge-relevance condition. However, for this condition, anterior HC showed an increase in SME in functional connectivity with the left MTG across the two time points. This increase was not present for the low knowledge-relevance condition (Table 2). However, the memory $\times$ time $\times$ condition analysis did not identify a cluster exceeding our significance criterion $(z>2.3$, cluster corrected). A follow-up analysis at a lowered voxel threshold ( $z>$ 1.96) that used an adjusted cluster correction to maintain the same cluster threshold $(p<0.05)$ revealed the hypothesized three-way interaction, specifically in the left MTG cluster identified for the high knowledge-relevance condition. Increases in HC-neocortical connectivity were observed for both high and low knowledge-relevance conditions in frontal and parietal regions as well (Table 2). Thus, to be more certain about the specificity of the connectivity increases between anterior HC and neocortical regions involved in semantic processing, further studies are needed. A potential next step could be to investigate whether the observed increased HC-neocortical connectivity persists during resting state. Activity in left MTG is consistently observed during the retrieval of conceptual knowledge, especially lexical-semantic knowledge, and lesions to this region lead to severe deficits in the retrieval of word meaning (Lau et al., 2008; Binder et al., 2009; Turken and Dronkers, 2011). The accumulation of knowledge might have facilitated episodic memory formation in our task specifically by enhancing neural communication between HC and association cortex, including MTG. Connectivity increases are often observed in training studies and have been proposed to reflect improved communication between brain areas (Büchel et al., 1999; Kelly and Garavan, 2005). In the memory domain, increased connectivity between $\mathrm{HC}$ and lateral temporal lobe regions is associated with successful memory formation (Gagnepain et al., 2011). Gagnepain et al. (2011) demonstrated that a decreased HC SME and increased hippocampalneocortical connectivity together were associated with the memory benefit due to priming. These findings underscore the importance of cortical inputs to the $\mathrm{HC}$, which may alter memory formation processes in the $\mathrm{HC}$. Therefore, the observed increase in connectivity between the HC and the left MTG in our study may reflect increased connections to nodes within the more extensive medical knowledge network, which in turn facilitated hippocampal binding of faces to diagnoses.

In contrast to previous studies pointing to the importance of $\mathrm{mPFC}$ for schema-related memory processing (van Kesteren et al., 2010, 2014; Warren et al., 2014; Brod et al., 2015), the mPFC did not show reliable condition differences in our study. However, we found tentative evidence for an increasing $\mathrm{mPFC}$ involvement for the high knowledge-relevance condition in the PPI analysis. While this finding is in line with claims about the $\mathrm{mPFC}$ biasing HC processing when prior knowledge is highly relevant, a bias that can be assumed to increase with increasing schema strength, it has to be treated with caution because of the lack of a significant memory $\times$ time $\times$ condition interaction. In general, the lack of a strong mPFC engagement in our task, which is apparently inconsistent with earlier studies, may reflect crucial differences among the tasks. In memory tasks, mPFC activation has been assumed to reflect the evaluation of new experiences based on their fit, or congruence, to schematic knowledge. When congruency is high, the $\mathrm{mPFC}$ is furthermore assumed to inhibit the HC (van Kesteren et al., 2012; Brod et al., 2013; Ghosh et al., 2014; Warren et al., 2014). The present task conditions, however, did not vary in congruency, but in relevance, as prior medical knowledge was highly relevant for diagnoses, but less so for names. Future studies should vary both congruency and relevance to obtain a more complete picture of mPFC contributions to schema-related modulations of episodic memory.

A potential concern in our design is the use of the same faces at the two testing sessions, which might have induced proactive interference at T2. Proactive interference is typically observed in pairedassociate cued recall when the cue has been previously associated with a different response. We used a forced choice recognition task in which the participants had to choose from either four names or four diagnoses (never mixed). Thus, even though the participants saw the T1 faces again 3 months later at T2, interference from the initial association was unlikely to be strong.

A strength of the present study is its high external validity. We examined how neural activation during the encoding of knowledge-relevant associative episodes changed as a function of increasing domain knowledge. Both the domain knowledge and 
its increase were not engineered in the laboratory, but resulted from future doctors preparing for their final medical exams. Thus, learning intensity and knowledge acquisition were beyond the scope of a laboratory experiment, but could be monitored closely via a web-based learning platform. In particular, performance on the learning platform was highly predictive of later exam success. The associative memory task consisted of encoding and later remembering face-name and face-diagnosis associations, and bore some resemblance to real-world memory situations that doctors encounter in their professional lives. The diagnoses were selected by medical professionals and carefully piloted to capture the assumed knowledge gains while preparing for the final medical exam. We observed that participants with greater knowledge gains showed greater decreases in right HC SME for knowledge-relevant pairs. Thus, we observed, for the first time, an association between changes in knowledge due to intensive learning in a real-world educational setting and changes in brain activation in a laboratory episodic memory paradigm (for related cross-sectional findings, see van Kesteren et al., 2014). This finding extends the literature on the effects of intensive, real-world studying on brain plasticity (Draganski et al., 2006) and connectivity at rest (Mackey et al., 2013) in that it uncovers changes in brain activation and connectivity in a transfer task and relates those to gains in content knowledge. Increments in content knowledge across 3 months of intensive study correlated with HC SME decrements for knowledge-relevant material. The knowledge gains were assessed using a web-based learning platform on which the participants studied for their final exam. We believe that combining laboratory-based cognitive neuroscience research with real-world educational settings and relating the two, in our case via educational technology, holds great promise for the study of memory in itself as well as for bridging the proclaimed gap between cognitive neuroscience and education (Ansari and Coch, 2006; Blakemore and Bunge, 2012). We suggest that future neuroscience research would profit from making greater use of knowledge acquisition in real-world contexts, such as schooling, vocational training, and the workplace, to better understand the neural pathways, areas, and mechanisms through which knowledge affects memory for new information in individuals of different ages.

\section{References}

Alba JW, Hasher L (1983) Is memory schematic? Psychol Bull 93:203-231. CrossRef

Ansari D, Coch D (2006) Bridges over troubled waters: education and cognitive neuroscience. Trends Cogn Sci 10:146-151.

Badre D, Wagner AD (2007) Left ventrolateral prefrontal cortex and the cognitive control of memory. Neuropsychologia 45:2883-2901. CrossRef Medline

Bartlett FC (1932) Remembering: an experimental and social study. Cambridge, UK: Cambridge UP.

Binder JR, Desai RH, Graves WW, Conant LL (2009) Where is the semantic system? A critical review and meta-analysis of 120 functional neuroimaging studies. Cereb Cortex 19:2767-2796. CrossRef Medline

Blakemore SJ, Bunge SA (2012) At the nexus of neuroscience and education. Dev Cogn Neurosci 2 [Suppl]:S1-S5.

Bransford J, Johnson M (1972) Contextual prerequisites for understanding: some investigators of comprehension and recall. J Verbal Learning Verbal Behav 11:717-726. CrossRef

Brewer JB, Zhao Z, Desmond JE, Glover GH, Gabrieli JD (1998) Making memories: brain activity that predicts how well visual experience will be remembered. Science 281:1185-1187. CrossRef Medline

Brod G, Werkle-Bergner M, Shing YL (2013) The influence of prior knowledge on memory: a developmental cognitive neuroscience perspective. Front Behav Neurosci 7:139. CrossRef Medline

Brod G, Lindenberger U, Werkle-Bergner M, Shing YL (2015) Differences in the neural signature of remembering schema-congruent and schemaincongruent events. Neuroimage 117:358-366. CrossRef Medline

Büchel C, Coull JT, Friston KJ (1999) The predictive value of changes in effective connectivity for human learning. Science 283:1538-1541. CrossRef Medline

Cohen J, Cohen P, West SG, Aiken LS (2003) Applied multiple correlation/ regression analysis for the behavioral sciences. New York: Routledge.

Davachi L (2006) Item, context and relational episodic encoding in humans. Curr Opin Neurobiol 16:693-700. CrossRef Medline

Draganski B, Gaser C, Kempermann G, Kuhn HG, Winkler J, Büchel C, May A (2006) Temporal and spatial dynamics of brain structure changes during extensive learning. J Neurosci 26:6314-6317. CrossRef Medline

Gagnepain P, Henson R, Chételat G, Desgranges B, Lebreton K, Eustache F (2011) Is neocortical-hippocampal connectivity a better predictor of subsequent recollection than local increases in hippocampal activity? New insights on the role of priming. J Cogn Neurosci 23:391-403. CrossRef Medline

Ghosh VE, Gilboa A (2014) What is a memory schema? A historical perspective on current neuroscience literature. Neuropsychologia 53:104-114. CrossRef Medline

Ghosh VE, Moscovitch M, Melo Colella B, Gilboa A (2014) Schema representation in patients with ventromedial PFC lesions. J Neurosci 34: 12057-12070. CrossRef Medline

Hickok G, Poeppel D (2007) The cortical organization of speech processing. Nat Rev Neurosci 8:393-402. CrossRef Medline

Kelly AM, Garavan H (2005) Human functional neuroimaging of brain changes associated with practice. Cereb Cortex 15:1089-1102. CrossRef Medline

Lau EF, Phillips C, Poeppel D (2008) A cortical network for semantics:(de) constructing the N400. Nat Rev Neurosci 9:920-933. CrossRef Medline

Liang JC, Wagner AD, Preston AR (2013) Content representation in the human medial temporal lobe. Cereb Cortex 23:80-96. CrossRef Medline

Mackey AP, Singley AT, Bunge SA (2013) Intensive reasoning training alters patterns of brain connectivity at rest. J Neurosci 33:4796-4803. CrossRef Medline

McClelland JL (2013) Incorporating rapid neocortical learning of new schema-consistent information into complementary learning systems theory. J Exp Psychol Gen 142:1190-1210. CrossRef Medline

Minear M, Park DC (2004) A lifespan database of adult facial stimuli. Behav Res Methods Instrum Comput 36:630-633. CrossRef Medline

O'Reilly JX, Woolrich MW, Behrens TE, Smith SM, Johansen-Berg H (2012) Tools of the trade: psychophysiological interactions and functional connectivity. Soc Cogn Affect Neurosci 7:604-609. CrossRef Medline

Paller KA, Wagner AD (2002) Observing the transformation of experience into memory. Trends Cogn Sci 6:93-102. CrossRef Medline

Piaget J (1952) The origins of intelligence in children. New York: International Universities.

Poppenk J, Evensmoen HR, Moscovitch M, Nadel L (2013) Long-axis specialization of the human hippocampus. Trends Cogn Sci 17:230-240. CrossRef Medline

Preston AR, Eichenbaum H (2013) Interplay of hippocampus and prefrontal cortex in memory. Curr Biol 23:R764-R773. CrossRef Medline

Prince SE, Daselaar SM, Cabeza R (2005) Neural correlates of relational memory: successful encoding and retrieval of semantic and perceptual associations. J Neurosci 25:1203-1210. CrossRef Medline

R Core Team (2014) R: a language and environment for statistical computing. Vienna, Austria: R Foundation for Statistical Computing.

Ranganath C, Ritchey M (2012) Two cortical systems for memory-guided behaviour. Nat Rev Neurosci 13:713-726. CrossRef Medline

Schlichting ML, Mumford JA, Preston AR (2015) Learning-related representational changes reveal dissociable integration and separation signatures in the hippocampus and prefrontal cortex. Nat Commun 6:8151. CrossRef Medline

Smith S, Jenkinson M, Woolrich M, Beckmann CF, Behrens TE, JohansenBerg H, Bannister PR, De Luca M, Drobnjak I, Flitney DE, Niazy RK, Saunders J, Vickers J, Zhang Y, De Stefano N, Brady JM, Matthews PM (2004) Advances in functional and structural MR image analysis and implementation as FSL. Neuroimage 23 [Suppl]:S208-S219. CrossRef Medline

Stanovich KE (1986) Matthew effects in reading: some consequences of individual differences in the acquisition of literacy. Read Res Q 21:360-407. CrossRef 
Tse D, Langston RF, Kakeyama M, Bethus I, Spooner PA, Wood ER, Witter MP, Morris RG (2007) Schemas and memory consolidation. Science 316:76-82. CrossRef Medline

Tse D, Takeuchi T, Kakeyama M, Kajii Y, Okuno H, Tohyama C, Bito H, Morris RGM (2011) Schema-dependent gene activation and memory encoding in neocortex. Science 333:891-895. CrossRef Medline

Turken AU, Dronkers NF (2011) The neural architecture of the language comprehension network: converging evidence from lesion and connectivity analyses. Front Syst Neurosci 5:1. CrossRef Medline

van Kesteren MT, Rijpkema M, Ruiter DJ, Fernández G (2010) Retrieval of associative information congruent with prior knowledge is related to increased medial prefrontal activity and connectivity. J Neurosci 30:1588815894. CrossRef Medline

van Kesteren MT, Ruiter DJ, Fernández G, Henson RN (2012) How schema and novelty augment memory formation. Trends Neurosci 35:211-219. CrossRef Medline

van Kesteren MT, Rijpkema M, Ruiter DJ, Morris RG, Fernández G (2014) Building on prior knowledge: schema-dependent encoding processes relate to academic performance. J Cogn Neurosci 26:2250-2261. CrossRef Medline

Wagner AD, Schacter DL, Rotte M, Koutstaal W, Maril A, Dale AM, Rosen
BR, Buckner RL (1998) Building memories: remembering and forgetting of verbal experiences as predicted by brain activity. Science 281 : 1188-1191. CrossRef Medline

Wang SH, Morris RG (2010) Hippocampal-neocortical interactions in memory formation, consolidation, and reconsolidation. Annu Rev Psychol 61:49-79, C1-4. CrossRef Medline

Warren DE, Jones SH, Duff MC, Tranel D (2014) False recall is reduced by damage to the ventromedial prefrontal cortex: implications for understanding the neural correlates of schematic memory. J Neurosci 34:76777682. CrossRef Medline

Weiskopf N, Hutton C, Josephs O, Deichmann R (2006) Optimal EPI parameters for reduction of susceptibility-induced BOLD sensitivity losses: a whole-brain analysis at $3 \mathrm{~T}$ and $1.5 \mathrm{~T}$. Neuroimage 33:493-504. CrossRef Medline

Zeithamova D, Dominick AL, Preston AR (2012) Hippocampal and ventral medial prefrontal activation during retrieval-mediated learning supports novel inference. Neuron 75:168-179. CrossRef Medline

Zimmer HD, Mecklinger A, Lindenberger U (2006) Handbook of binding and memory: perspectives from cognitive neuroscience. New York: Oxford UP. 